Int. J. Odontostomat.,

7(1):39-46, 2013.

\title{
Nivel de Conocimiento de Salud Oral y Utilización de GES Odontológico en Puérperas Atendidas en una Clínica Privada, Santiago, Chile
}

\author{
Oral Health Level of Knowledge and Use of Dental GES \\ in Puerperal Patients at a Private Clinic, Santiago, Chile
}

\author{
Juan Núñez; Patricia Moya*; María José Monsalves"*** \& Sylvia Landaeta M. ${ }^{* *+*}$
}

NÚÑEZ, J.; MOYA, P.; MONSALVES, M. J. \& LANDAETA, M. S. Nivel de conocimiento de salud oral y utilización de GES odontológico en puérperas atendidas en una clínica privada, Santiago, Chile. Int. J. Odontostomat., 7(1):39-46, 2013.

RESUMEN: El objetivo de este estudio fue conocer el nivel de conocimientos de salud oral y la utilización de GES odontológico en 200 puérperas atendidas en el servicio integral de la mujer de una clínica privada, del sector norte de Santiago de Chile durante el año 2011. Se aplicó un cuestionario auto-administrado compuesto de 24 preguntas relacionadas con el nivel de conocimiento sobre enfermedades orales, prevención, gestación, atención odontológica y desarrollo dentario. El punto de corte para ser considerado como buen nivel de conocimiento fue igual o mayor a 14 puntos y regular entre 7 y 13 puntos. La edad promedio de la muestra estudiada fue de 29,11 años $(+5,71)$ y la mitad refirió tener un solo hijo. El 57\% refiere educación universitaria, un 70,5\% declaró estar trabajando y sólo un tercio de ellas recibió atención dental durante su embarazo. Existe un nivel de conocimientos bueno en un $35 \%$ de las puérperas y regular en un $64 \%$, siendo los conocimientos sobre prevención en salud oral los mejores evaluados y el de crecimiento y desarrollo dentario los más deficientes. La edad media fue mayor en el grupo con mejor nivel de conocimientos. Al hacer el análisis estadístico según las categorías de la encuesta no se observó diferencias estadísticamente significativas entre el nivel de conocimientos y haber recibido atención dental durante el embarazo $(p=0,64)$. La relación entre el nivel de conocimientos sobre salud oral y paridad $(p=0,37)$, nivel educacional $(p=0,35)$ y ocupación $(p=0,39)$ no fueron estadísticamente significativas. Se analizó también el puntaje en comparación a las diferentes variables mediante regresión lineal múltiple, solo el nivel educacional de la madre (IC95\%= 0,3:1,8; $p=0.007)$ y la paridad $(I C 95 \%=-0,1: 1,5 ; p=0.05)$ fueron significativos, sin embargo el intervalo de confianza para la paridad no rechaza la hipótesis nula. Las madres con nivel educacional superior o técnico tiene un $4,6 \%$ más de conocimiento que las madres con nivel educacional inferior ( 1 punto de conocimiento; IC95\%= 0,3:1,8; $p=0.007$ ). En conclusión, el nivel de conocimiento de las puérperas resultó ser similar al de otros países latinoamericanos, sin embargo los valores encontrados distan de los observados en países desarrollados. Existe un número importante de mujeres embarazadas que no hacen uso de su garantía GES en atención dental. La educación en salud oral durante el control prenatal debe ser una tarea prioritaria de los servicios de salud, tanto a nivel de motivación como de formar hábitos saludables para la familia.

PALABRAS CLAVE: salud oral, puérperas, educación, GES.

\section{INTRODUCCIÓN}

La Promoción de la Salud es una de las estrategias propuestas por la OMS para incrementar en la población el control sobre la salud y mejorarla. El éxi- to dependerá del conocimiento previo de las pautas culturales y estilos de vida de la población objetivo (Almarales \& Llerandi, 2008).

\footnotetext{
Cirujano Dentista. Universidad Finis Terrae. Matrón clínico, Servicio de pabellón, Clínica Dávila. Universidad de Santiago de Chile, Santiago, Chile.

* Master en Salud Pública y Sistemas de Salud. Docente Facultad de Odontología. Universidad Finis Terrae, Santiago, Chile.

*** Cirujano Dentista. Docente Facultad de Odontología, Universidad Finis Terrae. (C) Magister en Epidemiología Clínica. Doctorando en Salud Pública, Universidad de Chile, Santiago, Chile.

Matrona clínica de servicio integral de la mujer, Clínica Dávila, Universidad de Santiago de Chile, Santiago, Chile. Este estudio fue realizado en el servicio integral de la mujer de una clínica privada en Santiago de Chile y patrocinado por la empresa Colgate Palmolive.
} 
En Chile, son escasos los estudios relacionados con conocimientos de salud oral en embarazadas y puérperas. Si bien es cierto, la educación y prevención en salud oral es preferentemente responsabilidad del odontólogo, esta acción debe involucrar a la familia, educando primero a los padres y transferir un adecuado conocimiento a sus hijos (Córdova \& Bulnes, 2007).

El conocimiento sobre salud oral que adquiere la mujer durante su vida es la piedra angular para el desarrollo de hábitos saludables y constituye una estrategia que permite promocionar actitudes favorables en salud a su entorno familiar. Una buena salud oral es un componente fundamental para el logro de una buena calidad de vida en las mujeres durante el embarazo y puerperio. Y por tanto para el recién nacido y el resto del grupo familiar (Saddki et al., 2010).

Para promover comportamientos saludables se debe tener en cuenta que las personas necesitan tener la información sobre qué hacer y cómo hacerlo. Esta información permite fomentar la aparición de nuevas creencias que la consideren, y que puedan ser incluidas a las tradiciones culturales de los grupos humanos. De aquí nace la necesidad de proporcionar educación sobre salud oral para las mujeres embarazadas durante el control prenatal a fin de destacar la importancia de una buena salud oral en el logro de una buena salud tanto para la madre y su bebé (Abiola et al., 2011).

La existencia de ciertas creencias y mitos populares relacionados con la salud oral durante y después del embarazo influyen en las actitudes que asumen las mujeres en el proceso salud enfermedad. Modificar la conducta individual de la embarazada con el fin de reducir el riesgo de enfermar o de aumentar la salud y el bienestar es el objetivo común de las diferentes concepciones de la salud pública (Fuentes et al., 2009).

Adicionalmente se concibe que toda creencia se desarrolla, transmite y mantiene a través de la experiencia del grupo social que la práctica, por lo cual las creencias durante el embarazo tienen gran ámbito de desarrollo en el contexto de familia (abuela-madreembarazada) y en el contexto social (embarazadaembarazada), en el cual el individuo está inserto, la información que se toma del contexto es procesada y utilizada de acuerdo a cada sujeto (Renfigo, 2009).

La evidencia muestra que mientras más precoz sea la prevención basada en un conocimiento sólido de salud oral en los progenitores, este conocimiento positivo por transferencia, será heredado a sus hijos, por lo que se traduce a nivel de salud pública en mejores índices de salud oral, ya sea en niños y a futuros en personas adultas (Cartes et al., 2009).

Existen múltiples factores que pueden estar interviniendo en el nivel de conocimientos y hábitos relacionados con la salud oral en las embarazadas y/o puérperas, entre ellos destacan factores sociodemográficos como la edad de la madre, nivel socioeconómico y de educación, ocupación, entre otras, como también la cultura médica-odontológica adquirida por indicaciones de autocuidado de los profesionales durante la atención en salud (Rodríguez, 2002; Garbero \& Delgado, 2005).

El autocuidado de la salud es una práctica que se adquiere en la medida en que las personas modifican su percepción del valor de la salud y su conceptualización de riesgo. La Reforma de Salud en Chile incluyó en las garantías explicitas en salud, (GES) "Salud Oral Integral de la embarazada" que tiene dentro de sus objetivos educar, prevenir, recuperar y rehabilitar la salud oral de la gestante, programa que se está realizando desde julio del 2009 y cuya responsabilidad recae en el profesional odontólogo (MINSAL, 2008). Este programa otorga acceso, oportunidad y protección financiera en la atención odontológica integral de la embarazada hasta nueve meses post- parto. Durante la atención odontológica, el odontólogo debe entregar información educativa respecto a hábitos saludables como instrucción sobre técnicas de cepillado, hábitos dietéticos y uso de fluoruros, así como respecto del consumo de tabaco y exposición al humo del mismo. También debe educar sobre organogénesis, formación de piezas dentarias, cuidados al recién nacido y lactante, y caries temprana de la niñez pensando en la salud del binomio madre-hijo. Sin embargo no se cuenta con información sobre conocimientos y prácticas en salud oral de las embarazadas en su control prenatal y/o puérperas en su control post-natal independiente de las aseguradoras de salud a la cual pertenezcan.

El propósito de este estudio fue conocer el nivel de conocimientos de salud oral y la utilización de los servicios odontológicos durante el control prenatal en puérperas del servicio integral de la mujer de una clínica privada del sector norte de Santiago de Chile, con el fin de diagnosticar y dirigir los esfuerzos hacia la prevención de la salud oral de la madre y de sus hijos. 


\section{MATERIAL Y MÉTODO}

Se realizó un estudio de corte transversal en un grupo de 200 puérperas que se atienden en el Servicio Integral de la Mujer de una clínica privada del sector norte de Santiago de Chile. Dentro de los criterios de exclusión se consideraron a aquellas puérperas que por problemas de tipo psicológicos/mentales o patológico no presentan las condiciones para responder el cuestionario autoadministrado. El Comité de Ética de la institución otorgó la autorización para realizar el estudio garantizando el anonimato y la privacidad de los resultados individuales. Las mujeres fueron invitadas a participar y contestar la encuesta en forma voluntaria, aplicando un consentimiento informado para cada participante.

Se recabaron datos sociodemográficos por medio de un formulario estandarizado. Las variables consideradas fueron edad, paridad, nivel educacional y ocupación. La ocupación se dicotomizó en ocupada o no ocupada, valor que se estimó mediante la variable proxy de aporte de ingresos económicos a la familia. Y el uso de los servicios odontológicos durante el control prenatal (esta variable fue incluida en el estudio, ya que fue referida en la literatura por su posible relación con un mejor conocimiento sobre salud oral en las embarazadas). El instrumento utilizado para medir conocimientos, actitudes y prácticas fue un cuestionario auto-administrado que consta de 22 preguntas (Martignon et al., 2008); que incluye ítems relacionados con prevención (siete), enfermedades orales (cinco), gestación en la atención odontológica (seis) y desarrollo dentario (cuatro). El grupo de preguntas sobre prevención en salud oral integró contenidos de medidas preventivas como cepillado dental, aplicación de flúor, alimentación e higiene. Para evaluar los conocimientos sobre enfermedades orales, se consultaron conceptos de gingivitis, enfermedad periodontal, caries y placa bacteriana. En cuanto a gestación y atención dental se preguntó sobre uso de anestesia, toma de radiografías dentales durante el embarazo y de atención odontológica pediátrica. En el desarrollo y crecimiento dentario, se incluyeron conceptos de dentición temporal como cronología de erupción, tipos de dentición y formación dentaria. Se consideró como buen conocimiento de salud oral un puntaje igual o superior a catorce puntos. Entre siete y trece puntos como regular conocimiento y menor a siete puntos como escaso conocimiento (puntos de corte referidos por la validación del instrumento para habla hispana).
La edad media de la muestra estudiada fue de 29,11 años (DE 5,71) en donde la edad mínima fue de 18 años y la edad máxima de 43 años. Un $50,5 \%$ de las puérperas reporta tener un sólo hijo, un $29 \%$ dos y un $14 \%$ tres hijos. Sólo un $6,5 \%$ refiere cuatro o más hijos. El $57 \%$ de las puérperas refiere tener educación universitaria y un $41 \%$ educación media. Un $70,50 \%$ de las encuestadas aporta ingresos a la familia por lo que se clasificó como ocupada (ver Tabla I).

Previa a la aplicación del cuestionario, se realizó un estudio piloto en 12 puérperas con el objetivo de detectar problemas en el vocabulario y conceptos. Ya que el instrumento esta validado para personas de habla hispana.

Para el análisis descriptivo, se calculó media, desviación estándar y proporciones con un intervalo de confianza del $95 \%$, según correspondiese. Se utilizó la prueba estadística T-test para establecer asociación entre nivel de conocimientos y las variables estudiadas. Para esta parte del análisis se trabajó la variable de resultado como variable continua ya que la categoría de bajo nivel fue subrepresentada en la muestra. Se analizaron los resultados de los puntajes en relación a las variables mediante regresión lineal múltiple (RLM) y se realizó análisis de residuos para evaluar el ajuste del modelo. La tabulación de los datos se realizó con el software MS Excel 2007 y el análisis estadístico con el programa STATA 12.0.

\section{RESULTADOS}

El 31\% $(n=62)$ de las puérperas refiere haber recibido atención dental durante el embarazo, siendo utilizado el sistema público por un $70,9 \%$ de ellas ( $n=$ 44). El nivel de conocimientos de salud oral fue clasificado como bueno en un $35 \%$ de las puérperas (media 30,18 años IC95\% 28,75: 31,61 ) y regular en un 64\% (media 28,6 años IC95\% 27,64: 29,56). No se observaron diferencias estadísticamente significativas entre el conocimiento y haber recibido atención dental durante el embarazo (IC95\% -1,22: 0,42; $p=0,33$ ). Lo mismo para la relación entre la ocupación (IC95\% 1,03: 0,$63 ; p=0,63$ ) y el conocimiento. Al analizar la relación entre el conocimiento sobre salud oral y la paridad es posible observar una tendencia al alza mientras es mayor el número de hijos, siendo esta diferen- 
cia estadísticamente significativa (IC95\% -1,74: -0,24; $\mathrm{p}=0,009)$. También se encontraron diferencias significativas para la relación con el nivel educacional (IC95\% $-1,84:-0,32 ; p=0,006)$ y la edad (IC95\% -1,87: $-0,35$; $p=0,005)$. Estos resultados se resumen en la Tabla I.

Al realizar el análisis de las variables mediante regresión lineal múltiple es posible ver que tanto el nivel socioeconómico como la paridad son estadísticamente significativas $(p=<0,05)$, sin embargo el IC de paridad no rechaza la hipótesis nula, por lo que sólo el nivel socioeconómico estaría relacionado con un mejor conocimiento de salud oral. Ya que las mujeres con nivel educacional técnico o superior pre- sentan 1,05 puntos más puntos de conocimiento que una mujer con nivel educacional básico o medio $(0,29$ : 1,83; $p=0,007)$, como se resume en la Tabla II. Para el análisis se graficaron los residuos (Fig. 1), y se utilizó el test de Shapiro-Wilk para evaluar normalidad, que arrojo una distribución normal de los residuos del modelo $(p=0,80)$.

Un $78,9 \%$ de las puérperas tiene conocimientos relacionados con prevención en salud oral, sin embargo son escasos los conocimiento sobre crecimiento y desarrollo dental de su hijo, como por ejemplo el saber cuando se forman y cuantos dientes tendrá su hijo $(30,1 \%)$.

Tabla I. Relación entre el conocimiento en salud oral y las variables estudiadas.

\begin{tabular}{|c|c|c|c|c|}
\hline Variable & Categoría & Promedio & IC 95\% & $\mathbf{p}$ \\
\hline Atención odontológica & Recibió atención & 12,29 & $11,6: 13,0$ & \\
\hline \multirow[t]{2}{*}{ durante el embarazo } & No recibió atención & 12,70 & $12,23: 13,16$ & \\
\hline & Diferencia & $-0,41$ & $-1,22: 0,42$ & 0,33 \\
\hline \multirow[t]{3}{*}{ Ocupación } & Con ocupación & 12,51 & $12,04: 12,98$ & \\
\hline & Sin ocupación & 12,71 & 12,06: 13,36 & \\
\hline & Diferencia & $-0,20$ & $-1,03: 0,63$ & 0,63 \\
\hline \multirow[t]{3}{*}{ Paridad } & Un hijo & 12,08 & $11,57: 12,60$ & \\
\hline & Dos o más hijos & 13,07 & $12,52: 13,63$ & \\
\hline & Diferencia & $-0,99$ & $-1,74:-0,24^{*}$ & $0,009^{*}$ \\
\hline \multirow[t]{3}{*}{ Nivel educacional } & Básica o media & 11,94 & 11,33: 12,55 & \\
\hline & Técnica o superior & 13,01 & $12,54: 13,5$ & \\
\hline & Diferencia & $-0,77$ & $-1,84:-0,32^{*}$ & $0,006^{*}$ \\
\hline \multirow[t]{3}{*}{ Edad } & Menor o igual a 30 & 12,11 & 11,62: 12,60 & \\
\hline & Mayor de 30 años & 13,21 & $12,62: 13,80$ & \\
\hline & Diferencia & $-1,10$ & $-1,87:-0,34^{*}$ & $0,005^{*}$ \\
\hline
\end{tabular}

* $p$ estadísticamente significativo; * IC95\% consistente; rechaza hipótesis nula.

Tabla II. Coeficientes de regresión lineal múltiple del puntaje de conocimiento en salud oral en relación a las variables estudiadas.

\begin{tabular}{lccc}
\hline Variable & Coeficiente & IC 95\% & p \\
\hline Atención odontológica durante el & 0,13 & $-0,68: 0,94$ & 0,75 \\
Ocupación & 0,45 & $-0,37: 1,2$ & 0,28 \\
Paridad & 0,77 & $-0,11: 1,56$ & $0,05^{*}$ \\
Nivel educacional & 1,05 & $0,28: 1,83$ & $0,007^{*}$ \\
Edad & 0,74 & $-0,66: 1,55$ & 0,072 \\
\hline
\end{tabular}

* p estadísticamente significativo; * IC95\% consistente; rechaza hipótesis nula 


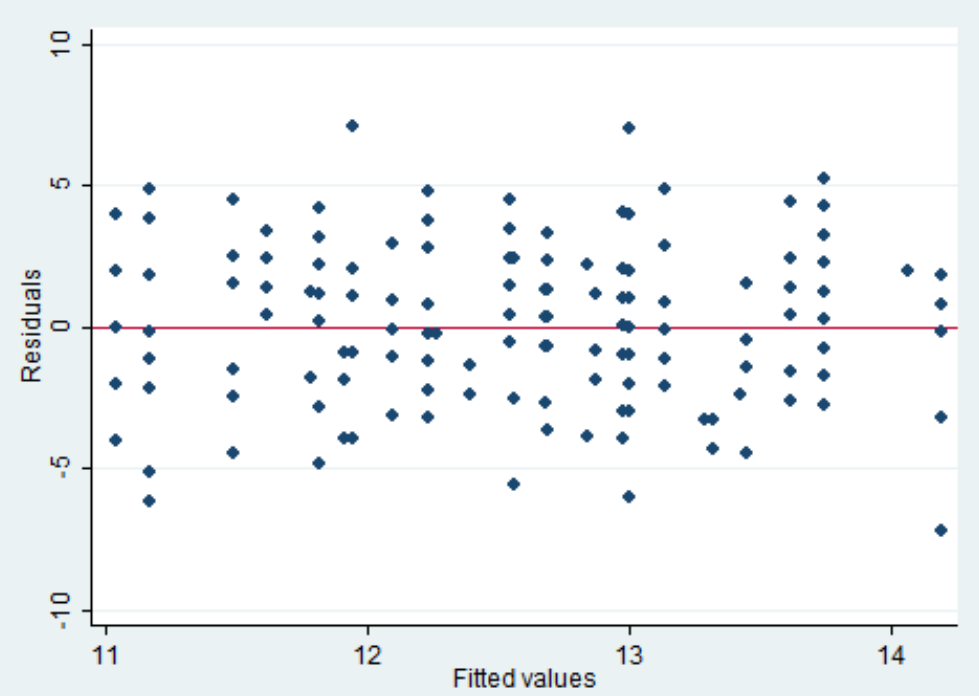

Fig. 1. Distribución de los residuos.

\section{DISCUSIÓN}

La mayoría de las patologías que provocan más morbi-mortalidad tienen relación con el nivel de educación y hábitos de la población, lo cual confirma una vez más el hecho de que los estilos de vida son uno de los determinantes principales de la salud en los países. La promoción de hábitos saludables es una de las medidas que más y mejor puede contribuir a la mejora de la esperanza y calidad de vida de la gente.

En Chile y en varios países del mundo existen programas con actividades específicas dirigidas a grupos prioritarios o de riesgo como son las mujeres embarazadas y cuyo objetivo principal es mejorar la salud oral junto con educar sobre el cuidado de la salud propia y la de sus hijos (Saddki et al.; Cartes et al.; MINSAL). Sin embargo, las mujeres encuestadas en nuestro estudio presentaron escasos conocimientos sobre la salud oral, así como de los procedimientos que el odontólogo puede realizar durante su embarazo.

El nivel de conocimiento sobre salud oral en las puérperas resultó ser similar a otros países latinoamericanos (Garbero \& Delgado; Rodríguez; Rengifo). Sin embargo, estos valores distan de los resultados que se obtienen en países desarrollados, como Estados Unidos, donde el conocimiento y prevención tiene un mayor enfoque en salud pública (Shein et al., 1999).

El nivel de conocimiento sobre salud oral fue regular en el $64 \%$ de las mujeres encuestadas, a pesar de que más de la mitad de ellas refiere un nivel educacional universitario (57\%). Estos valores son similares a los reportados por Córdova \& Bulnes que estiman un $66 \%$ y Rodríguez que refiere un $60 \%$ para la misma categoría. Estos valores establecen que no existe una relación significativa entre el conocimiento de salud oral y el nivel educacional de la madre, ya que como se puede ver en los resultados la diferencia es estadísticamente significativa ( $p<0,05$ y IC95\%) pero no clínicamente, ya que el mayor nivel educacional aumentaría solo 1 punto de conocimiento, como se observa en la RLM, lo que no nos habla de un nivel de conocimiento mayor (Almarales \& Llerandi; Keirse \& Plutzer, 2010). Para las otras variables es posible ver que a pesar que existe una tendencia de mayor conocimiento de salud oral a medida que es mayor la paridad y la edad, el aumento no es significativo en la RLM.

Existe un desconocimiento en general de la inocuidad de los procedimientos odontológicos por parte de las embarazadas lo que podría impedir la búsqueda de atención a sus problemas orales. Y Las razones citadas como barreras para buscar los servicios de salud oral incluyen el miedo y la ansiedad del tratamiento, la baja percepción de los problemas dentales y el tratamiento, y los conceptos erróneos acerca de los efectos de los tratamientos dentales en el desarrollo del feto (Rengifo, 2009; Almarales \& Llerandi; Fuentes et al.). Esto muestra la larga tradición de erróneos conceptos con respecto a la salud oral y sus cuidados durante el embarazo reportados (Ozen et al., 2012). En nuestro estudio esta visión se ve reflejada por el nivel regular del conocimiento en relación a la salud oral y el porcentaje que no recibe atención dental (69\%), a pesar de ser una patología cubierta por el GES en Chile.

En cuanto a la utilización de los servicios odontológicos durante el embarazo y/o puerperio, resulta alarmante observar que sólo el $31 \%$ de las encuestadas recibieron atención dental, ya sea en el sector privado o público de salud. Un estudio realizado en embarazadas entre 18 a 44 años de edad que reciben atención prenatal en un hospital universitario de Nigeria (LASUTH) reportó que un 33,0\% visitaron al dentista durante el embarazo actual sien- 
do la razón más común el dolor $88 \%$ (Adeniyi et al., 2010). Otro estudio realizado en 649 nulíparas que asisten a control prenatal en clínicas públicas en Adelaida, Australia del Sur, señala que sólo el $35 \%$ tuvo atención dental durante el embarazo, un 35\% no tenía ninguna visita al dentista por lo menos durante dos años y el $27 \%$ informó de costo como un importante elemento de disuasión (Keirse \& Plutzer). En la Encuesta Nacional de Salud de Chile 2009-2010 (ENS 2009-2010) se estimó que el $4,3 \%$ de la población no ha visitado nunca el odontólogo, el $23,5 \%$ no lo visita hace más de 5 años, el $28,2 \%$ no lo hace entre 1 y 5 años, el $20,4 \%$ lo visito entre 6 meses y 1 año y solo el 23,6\% hace menos de 6 meses (MINSAL \& Departamento Salud Pública PUC, 2010). Es interesante ver que en la ENS 2009-2010 si existe una gradiente educacional marcada. Esto puede deberse a que las características en población general difieren de las de grupos específicos, como en nuestro estudio. Principalmente asociado al desconocimiento general y las barreras culturales que existen en relación a la atención dental en embarazadas, como se indica en párrafos anteriores. A esto se suma que a pesar de ser una patología GES que podría ser demandada esta subutilizada, algunos autores han referido que la baja utilización de las prestaciones garantizadas puede deberse entre otros factores a la condición de ser atención ambulatoria y al desconocimiento de la cobertura (Fernández, 2006).

Del total de puérperas que refiere haber recibido atención dental durante el embarazo (31\%) un $70,9 \%$ se atendió en el sistema público. A pesar que todas las mujeres del presente estudio se encontraban bajo control en el servicio de la mujer de una clínica privada, la mayoría de las que utilizaron su garantía de salud dental lo hicieron en atención pública. Esto nos muestra que a pesar que las mujeres se atienden en el sistema privado para atención médica, no sucede lo mismo para atención odontológica. Hay varias razones que pueden explicar estos resultados, una hipótesis es el desconocimiento de las mujeres del uso de su garantía mediante Isapres, por lo que para estudios posteriores sería importante considerar esta variable dentro del análisis, lo que permitiría saber si las que no hacen uso de su garantía son aquellas adscritas a este tipo de previsión. Otra posibilidad es que las pacientes FONASA, pueden acceder a atención médica en instituciones privadas, mediante la libre elección, no así para la atención dental. Lo más probable es que ambas situaciones estén produciendo esta subutilización del GES de embarazadas, sumado al desconocimiento y mitos en relación a la inocuidad del tratamiento odontológico en embarazadas.
En cuanto a la utilización de los servicios odontológicos y su relación con el nivel de conocimiento de las mujeres encuestadas, la diferencia no fue estadísticamente significativa, evidenciando una posible relación con una educación deficiente por parte del odontólogo en el ámbito de salud oral, o la falta de énfasis en la educación en las embarazadas, por parte del mismo colectivo profesional.

Varios autores indican que el autocuidado de la salud es una práctica que se adquiere en la medida en que las personas modifican su percepción del valor de la salud, y que debe adquirirse desde niños (Fuentes et al.; Garbero \& Delgado; Ozen et al.). En el presente estudio, se evidencia el escaso conocimiento sobre la salud oral en las mujeres puérperas y la baja utilización de los servicios odontológicos durante el embarazo. Se esperaría que ambas variables estuviesen relacionadas, pero como se pudo pesquisar en este estudio, no hay una relación estadísticamente significativa. Lo que permite concluir que a pesar de acceder a atención odontológica durante el embarazo el conocimiento en salud oral no mejora.

Como corolario final es importante destacar la necesidad de evaluar el conocimiento de nuestros pacientes en relación a salud oral. En este grupo específico es aún más significativo, ya que al empoderar a la madre en el autocuidado, se hace en forma indirecta también en el niño, fortaleciendo el binomio madre- hijo. Por otra parte, se debe destacar el bajo número de mujeres que accede a atención dental a pesar que desde el año 2009 este grupo corresponde a priorización nacional, producto de su incorporación al GES. Es relevante visualizar este problema con una perspectiva desde la Salud pública, para identificar las razones de la baja utilización de esta garantía de salud. Parece necesario profundizar en el estudio del porqué de estos resultados, y estudiar la utilización de esta garantía en el sector público, que permita la comparación y la comprensión del escenario en su totalidad. Una alternativa para este grupo sería reforzar las estrategias en forma intersectorial, que permitan abordar en forma eficiente este problema, por medio de interconsultas para el uso de la garantía desde otros profesionales. Finalmente, nos parece necesario y recomendable reforzar conocimientos y buenas prácticas durante la realización del tratamiento, y no solo limitarnos a una educación inicial, para que los contenidos que pretendemos inculcar en los pacientes se mantengan como hábitos en el tiempo. Pudiendo intervenir realmente en el ciclo vital tanto de la madre como del hijo. 
Declaración de conflictos de intereses. No existe conflicto de interés con la empresa Colgate Palmolive. Su patrocinio se debe al premio obtenido por el alumno Juan Nuñez, coordinado entre la Facultad de Odontología de la Universidad Finis Terrae y dicha empresa, en el concurso "Colgate apoya tu tesis", buscando apoyar la investigación de alumnos de pregrado. Durante el estudio no se hizo alusión, ni promoción a la empresa ni sus productos, tal como establecen las bases del concurso.

NÚÑEZ, J.; MOYA, P.; MONSALVES, M. J. \& LANDAETA, M. S. Oral health level of knowledge and use of dental GES in puerperal patients at a private clinic, Santiago, Chile. Int. J. Odontostomat., 7(1):39-46, 2013.

ABSTRACT: The aim of this study was to determine the level of oral health knowledge in 200 postpartum women attending an integral service for women of a private clinic in the northern sector of Santiago de Chile during the year 2011. We used a self-administered questionnaire based on 24 questions related to the level of knowledge of oral diseases, prevention, pregnancy, dental care and dental development. The cutoff score for being considered a good level of knowledge was higher than or equal to 14 points and regular cooperation between 7 and 13 points. The average age of the sample was 29.11 years (+ 5.71 ) and half reported having one child. $57 \%$ refers university education, $70.5 \%$ reported to be working and only a third received dental care during pregnancy. Thirty-five percent of puerperal women had a good level of knowledge and $64 \%$ had a regular level. The highest score identified was the knowledge on prevention in oral health and the lowest was health growth and development of dental. The mean age was higher in the group with higher level of knowledge. No statistically significant difference was observed between the level of knowledge and having received dental care during pregnancy $(p=0.635)$. The relation between level of oral health knowledge and parity $(p=0.367)$, educational level $(p=0.345)$ and occupation $(p=0.388)$ was not statistically significant. Using linear regression we analyzed the relation between level of knowledge score and other variables. The level of education of the mother $(95 \% \mathrm{Cl}=0.3: 1.8, \mathrm{P}=0.007)$ and parity $(95 \% \mathrm{Cl}=-0.1: 1.5, \mathrm{p}=0.05)$ were significant, but the confidence interval for parity does not reject the null hypothesis. Mothers with higher or technical level of education had $4.6 \%$ increased knowledge than mothers with lower level of education ( 1 point of knowledge, $95 \% \mathrm{Cl}=0.3: 1.8, \mathrm{P}=0.007$ ). In conclusion, the level of knowledge of puerperal women was found to be similar to other Latin American countries, however the values found are far from those observed in developed countries. A significant number of pregnant women do not make use of their GES warranty for dental attention. Oral health education during prenatal care should be a priority for health services, both in terms of motivation as to promote healthy habits for the family.

KEY WORDS: oral health, puerperal women, education, GES.

\section{REFERENCIAS BIBLIOGRÁFICAS}

Abiola, A.; Olayinka, A.; Mathilda, B.; Ogunbiyi, O.; Modupe, S. \& Olubunmi, O. A survey of the oral health knowledge and practices of pregnant women in a Nigerian teaching hospital. Afr. J. Reprod. Health, 15(4):14-9, 2011.

Adeniyi, A.; Ogunbanjo, B.; Sorunke, M.; Onigbinde, O.; Agbaje, M. \& Braimoh, M. Dental attendance in a sample of Nigerian pregnant women. Nig. Q. J. Hosp. Med., 20(4):186-91, 2010.

Almarales, C. \& Llerandi, Y. Conocimientos sobre salud bucal y demanda de servicios estomatológicos en relación con la enfermedad periodontal en embarazadas. Rev. Cubana Estomatol., 45(2):0-0, 2008.

Cartes, R.; Mardones, S. \& Paredes, C. Conocimientos y estado de salud bucal en madres beneficiarias del sistema Chile Crece Contigo. Rev. Chil. Salud Pública, 13(3):136-42, 2009.

Córdova, J. \& Bulnes, R. Nivel de conocimiento sobre prevención de salud bucal en gestantes, hospital de la mujer, Villahermosa, Tabasco 2007. Horizonte Sanitario, 6(2):18-25, 2007.
Fernández, M. R. ¿GES o no GES? Esa es la cuestón. Rev. Esp. Econ. Salud, 5(6):361-8, 2006.

Fuentes, R.; Oporto, G.; Alarcón, A.; Bustos, L.; Prieto, R. \& Rico, $\mathrm{H}$. Opiniones y creencias de embarazadas en control prenatal relacionadas con salud oral y embarazo. Av. Odontoestomatol., 25(3) :147-54, 2009.

Garbero, I. \& Delgado A. Oral health in pregnant: knowledge ant attitudes. Acta Odontol., 43(2):135-40, 2005.

Keirse, M. J. \& Plutzer, K. Women's attitudes to and perceptions of oral health and dental care during pregnancy. J. Perinat. Med., 38(1):3-8, 2010.

Martignon, S.; Bautista, G.; González, M.; Lafaurie, G.; Morales, V. \& Santamaría, R. Instrumentos para Evaluar Conocimientos, Actitudes y Prácticas en Salud Oral para Padres/Cuidadores de Niños Menores. Rev. Salud Pública, 10(2):308-14, 2008.

MINSAL. Guía Clínica Atención odontológica integral de la embarazada. Santiago, Chile, Ministerio de Salud, 2008. 
NÚÑEZ, J.; MOYA, P.; MONSALVES, M. J. \& LANDAETA, M. S. Nivel de conocimiento de salud oral y utilización de GES odontológico en puérperas atendidas en una clínica privada, Santiago, Chile. Int. J. Odontostomat., 7(1):39-46, 2013.

MINSAL \& Departamento Salud Pública PUC. Segunda Encuesta Nacional de Salud 2009- 2010. Santiago, Chile, Ministerio de Salud, 2010.

Ozen, B.; Ozer, L.; Basak, F.; Altun, C. \& Acikel, C. Turkish Women's Self-Reported Knowledge and Behavior towards Oral Health during Pregnancy. Med. Princ. Pract., 21(4):318-22, 2012

Rengifo, $\mathrm{H}$. Creencias acerca de la salud oral en gestantes en tres ciudades colombianas. Rev. Fac. Odontol. Univ. Antioq., 20(2):171-8, 2009.

Rodríguez, M. Nivel de conocimiento sobre prevención en salud bucal en gestantes del Hospital Nacional Daniel A. Carrión en el 2002. Universidad Nacional Mayor de San Marcos, Perú, 2002.

Saddki, N.; Yusoff, A. \& Hwang, Y. L. Factors associated with dental visit and barriers to utilisation of oral health care services in a sample of antenatal mothers in Hospital Universiti Sains Malaysia. BMC Public Health, 10:75, 2010.

Shein, B.; Tsamsouries, A. \& Rovero, J. Seff reported compliance an the effectiveness of prenatal dental education. J. Clinic. Pediatr. Dent., 15(2):102-9, 1991.
Dirección para Correspondencia a:

Patricia Moya

Facultad de Odontología

Universidad Finis Terrae

Avda. Pedro de Valdivia 1509

Providencia

Santiago

CHILE

Tel: 93595419

Email: drapatriciamoya@yahoo.com

Recibido: 13-09-2012

Aceptado: 07-02-2013 